\title{
Symposium
}

\section{Lokales Wissen und Kulturvergleich im 21. Jahrhundert}

Symposiumsbeitrag zu: Max Haller (unter Mitarbeit von Anja Eder), Ethnic Stratification and Economic Inequality around the World. The End of Exploitation and Exclusion? Farnham: Ashgate 2015, 397 S., gb., 113,00€

Besprochen von Prof. Dr. Boike Rehbein: Kultur-, Sozial- und Bildungswissenschaftliche Fakultät, Humboldt-Universität zu Berlin, E-Mail: rehbeinb@hu-berlin.de

DOI 10.1515/srsr-2017-0004

Schlüsselwörter: Einkommensungleichheit, Ethnizität, Ländervergleich

Das Buch sucht die These zu belegen, dass ökonomische Ungleichheit nicht nur durch die Existenz sozialer Klassen, sondern auch durch Unterschiede zwischen ethnischen Gruppen innerhalb heutiger Nationalstaaten verstärkt werde. Daraus folge, dass ethnisch homogene Staaten statistisch einen geringeren Grad ökonomischer Ungleichheit aufwiesen. Ökonomische Ungleichheit wird im Buch fast ausschließlich als Einkommensungleichheit betrachtet. Als Ethnizität fasst der Autor jeden Unterschied, der mit Sprache, Religion und „,biosozialen“ Faktoren (gemeinsame Abstammung und Abgrenzung) verknüpft ist. Die These wird in erster Linie auf der theoretischen Grundlage der Soziologie Max Webers bearbeitet und an Hand verfügbarer Sekundärdaten belegt. Der erste Teil des Buches entwickelt den theoretischen Rahmen, die Methodologie und ein allgemeines Modell. Der zweite Teil enthält Studien einzelner Länder. Ein normativ ausgerichtetes Kapitel mit Handlungsempfehlungen, in erster Linie für die Politik, schließt das Buch.

Es ist sehr erfreulich, dass der Autor einige seiner Voraussetzungen transparent macht und dabei sogar die Wissenschaftstheorie skizziert, auf die er sich stützt. Im Anschluss an den Neukantianismus unterscheidet er idiografisches und nomothetisches Vorgehen (53). Wie Weber schlägt der Autor vor, beide Ansätze miteinander zu verbinden. Und in ähnlicher Weise führt er das Verstehen zwar als zentralen Begriff ein, bezieht es aber in die weitere Untersuchung nicht ein. Der Autor verbindet vielmehr ganz im Sinne Webers eine Reihe von Faktoren zu einem Geflecht, das auf S. 58 schematisch dargestellt ist. Die Faktoren werden so definiert und untersucht, dass sie in probabilistischer Weise einige Idealtypen eth- 
nischer Differenzierung erklären können. Die Weiterentwicklung der Wissenschaftstheorie seit Weber wird nur einbezogen, insoweit sie sich auf ihn stützt.

Der Autor verbindet mit seiner Wissenschaftstheorie eine methodische Vorgehensweise, die ebenfalls an Weber erinnert. Er identifiziert quantitative Methoden und nomothetisches Vorgehen sowie qualitative Methoden und idiografisches Vorgehen, aber die qualitative Methode beschränkt sich faktisch wie bei Weber auf das Studium der Literatur. Qualitative Comparative Analysis und die Fuzzy Set Analysis werden zu qualitativen Methoden erklärt. Den Einsatz dieser Instrumente halte ich im Hinblick auf das Ziel des Buches für sehr sinnvoll. Hier geht der Autor auch klar über Weber hinaus. Das quantitative Instrument der Wahl ist in erster Linie die Regressionsanalyse.

Die Instrumente werden auf Sekundärdaten angewandt. Der Autor wirft der deutschen Ungleichheitsforschung die Tendenz vor, ohne Daten zu arbeiten (9, Fußnote) und führt ausgerechnet Peter A. Berger und Anja Weiß als Beispiele dafür an. Da es unwahrscheinlich ist, dass der Autor einen Witz in sein Buch einbauen wollte, kann ich mir den Vorwurf nur vor dem Hintergrund der methodologischen Vorgehensweise erklären. Für den Autor ist empirische Forschung nach dem Vorbild Webers möglich, ohne eigene Daten zu erheben. Noch unnötiger scheint für den Autor zu sein, Feldforschung zu betreiben oder die Länder, über die er schreibt, auch nur besucht zu haben. Statt dessen verwendet er mit Bezug auf die ethnische Heterogenität vor allem Daten aus dem Fischer Weltalmanach, The Statesman's Yearbook und Wikipedia (96, Fußnote).

Vor diesem Hintergrund kann der Beitrag des Buches zur Forschung klarer gefasst werden. Da die empirischen Ergebnisse auf zweifelhaften Daten beruhen, halte ich das allgemeine Modell von elf Idealtypen (136, 164f.), das der Autor vorstellt, für problematisch. Ich erinnere daran, dass das Modell auf Einkommen basiert und andere Dimensionen ökonomischer Ungleichheit nicht berücksichtigt. (Der Autor weist selbst darauf hin, dass sein Musterbeispiel für die Korrelation von ethnischer Homogenität und Einkommensgleichheit, Schweden, im Hinblick auf die Vermögensverteilung eines der ungleichsten Länder der Welt ist.) Das Modell kann jedoch eine erste Annäherung an das Problem darstellen. So scheint der Autor es auch zu interpretieren. Die Fallstudien sind noch problematischer, wie ich weiter unten argumentieren werde, während die theoretischen und methodologischen Erwägungen letztlich eine Interpretation Max Webers darstellen. Der Autor führt außerdem als zentralen Beitrag seines Buches an, dass es gegen den Rest der Soziologie die Relevanz des Nationalstaates für das Verständnis ökonomischer Ungleichheit aufzeige, aber das kann nicht als Ergebnis gelten, weil mir niemand bekannt ist, der das leugnen würde.

Die zentrale These, die der Anfang dieser Rezension zusammenfasst, wirkt ebenso problematisch wie das Modell. Der Autor subsumiert Phänomene wie 
Race, Religionszugehörigkeit, sprachliche Unterschiede und zahlreiche Formen konstruierter Diskriminierung unter den Begriff der Ethnizität. Auch wenn diese Definition eigenwillig ist und dazu führt, dass der Autor in Kommentaren zu angelsächsischen Diskussionen das Wort „race“ meist einfach durch „ethnicity“ ersetzt, spricht aus wissenschaftlicher Sicht nichts gegen einen derartigen Versuch. (In politischer Hinsicht ist das etwas anders, wie ich unten argumentieren werde.) Ich halte es hingegen für wissenschaftlich fragwürdig, dass der Autor zwar Ethnizität durch Sprache, Religion und biosoziale Merkmale definiert, diese aber nicht eingehender diskutiert, operationalisiert und empirisch untersucht (31f.). Vielmehr werden in den Länderstudien Grenzen zwischen Sprachgruppen oder Religionen einfach aus der benutzten Literatur übernommen.

Das Beispiel Indiens zeigt, dass der Verlass auf sekundäre Daten in die Irre führt. Zunächst erklärt der Autor die ökonomische Ungleichheit in Indien mehrmals für sehr gering, weil der Gini-Wert im internationalen Maßstab niedrig sei. Wer auch nur einen Tag in Indien verbracht hat, wird dieser Aussage nicht zustimmen können. Ferner liegt die Vermögensungleichheit in Indien deutlich höher als die Einkommensungleichheit. Einige der reichsten und viele der ärmsten Menschen leben in Indien. Der Autor erklärt die vermeintlich geringe ökonomische Ungleichheit durch das Kastensystem. Für die Analyse des Kastensystems stützt er sich fast ausschließlich auf Max Weber und Louis Dumont. Während Weber nur begrenzte Quellen zur Verfügung hatte, ist heute nahezu jede Aussage Dumonts theoretisch und empirisch zweifelhaft (Jodhka, 2013). Beide Autoren basierten ihre Interpretationen auf schriftliche Darstellungen, die zumeist von Brahmanen angefertigt wurden, also aus einer bestimmten sozialen Perspektive. Ferner deuteten sie das Kastensystem so, wie es sich ihnen historisch darbot. Als solches war das System ein Konstrukt der englischen Volkszählung (Banerjee-Dube, 2008). In dieser Form hat es vor der Kolonialzeit nie existiert, auch wenn es sie im 20. Jahrhundert beeinflusste. Die kolonialistische Deutung des Kastenwesens führt bei Haller zu einer verzerrten Erklärung. Die Wirklichkeit des Kastensystems in Indien ist keineswegs durch Kooperation und horizontale Differenzierung geprägt, wie er argumentiert (215f.), sondern oft durch Extreme von Gewalt, die jenseits des Vorstellbaren liegen (Shah, 2002; Shah et al., 2006). Der Hinduismus und die Lehre der Reinheit spielen in dieser Wirklichkeit keineswegs die wichtige Rolle, die Weber, Dumont, die Brahmanen und Haller ihnen zuschreiben.

Der Autor hätte eine deutlich bessere Interpretation Indiens vorlegen können, wenn er die indische Soziologie stärker berücksichtigt hätte. Die wenigen vom Autor verwendeten indigenen Analysen stammen von Soziologen, die wegen ihrer parsonianischen Ausbildung in der Tradition Max Webers stehen (Srinivas, Gupta). Ein aktuelles Werk über die Diskussionen um den Kastenbegriff hätte ihn vor 
zahlreichen Irrtümern bewahrt (beispielsweise Jodhka, 2013). Die Irrtümer scheinen mir allerdings im wissenschaftstheoretischen Ansatz zu wurzeln. Der Autor schreibt zwar Sprache und Religion zentrale Rollen zu, beherrscht aber offensichtlich keine indische Sprache und hat keine empirische sowie nur geringe sekundäre Kenntnis indischer Religionen. Man mag erwidern, wie er es an mehreren Stellen andeutet, dass eine genaue Kenntnis der behandelten Länder über den Rahmen eines Überblickwerkes hinausgehe. Darauf würde ich antworten, dass der Autor sich auf die Länder hätte konzentrieren können, mit denen er vertraut ist, oder etwas mehr Zeit hätte investieren müssen.

Die Fallstudie Brasiliens, die in ein Kapitel zu Lateinamerika eingebettet ist, zeigt, dass die Kapitel zu Ländern, mit denen der Autor besser vertraut ist, auch besser gelungen sind. Erstens berücksichtigt der Autor die aktuellen Diskussionen in Brasilien stärker als im Falle Indiens. Zweitens behandelt er ausführlich die Rolle des Kolonialismus bei der Herstellung einer „farbigen Klassenstruktur“, während dieser Punkt im Falle Indiens völlig fehlt. Die Fallstudie Brasiliens kommt im Hinblick auf die Klassenstruktur zu Ergebnissen, die unseren Untersuchungen zu sozialer Ungleichheit ähneln. Unser Vorgehen, in einer multikulturellen Gruppe in der jeweiligen Landessprache $\mathrm{zu}$ forschen, deren Mitglieder sich teilweise auf mehrere Jahrzehnte Forschungserfahrung im Land stützen können, wäre übrigens auch eine Möglichkeit, die empirischen Probleme einer Überblicksforschung wie der Hallers zu lösen (Rehbein / Souza, 2014; Jodhka / Rehbein / Souza, 2017).

Dass dem Autor auch im Falle Brasiliens zahlreiche Irrtümer unterlaufen, halte ich für entschuldbar, selbst wenn fast alle durch eine Zusammenarbeit mit lokalen Wissenschaftlern vermeidbar gewesen wären. (Ein Beispiel ist die fehlerhafte Verwendung unseres Begriffs der ralé, 239.) Viel wichtiger ist die Stärke des Kapitels, die mit der Berücksichtigung des Kolonialismus verknüpft ist. Der Autor will in seinem Buch zeigen, dass ethnische Homogenität statistisch mit einer geringeren Einkommensungleichheit korreliert. Er kommt zum Ergebnis, dass diese Bedingungen vor allem in Europa gegeben seien und führt das auf das Alter des Nationalstaates zurück. Er erwähnt auch, dass die Homogenisierung ein staatliches und häufig gewaltsames Projekt gewesen sei. Dass die Heterogenität der Nationalstaaten im globalen Süden nicht allein auf ihrem geringen Alter, sondern auf dem Kolonialismus beruht, kommt im Buch hingegen nur im Kapitel über Brasilien voll zum Tragen. Mit Bezug auf Indien wird es gar nicht erwähnt was auf der orientalistischen Vorstellung Webers und Dumonts beruht, dass es auch vor der Kolonialzeit einen Staat Indien gegeben hätte.

So erscheinen ethnische Vielfalt und Staatsgrenzen im Buch als gegeben, ebenso wie Grenzen zwischen Sprachen und Religionen. Der Autor verarbeitet Daten über sprachliche und religiöse Zugehörigkeit, ohne sie kritisch zu hinter- 
fragen. Ein Blick auf Laos erläutert die Problematik (auch zu Laos siehe Rehbein / Souza, 2014). Offiziell werden im Nationalstaat Laos 48 Sprachen gesprochen. Einige Zählungen kommen jedoch auf rund 150. In ähnlicher Weise sind offiziell Buddhismus und einige andere Hochreligionen als Religionen anerkannt, Animismen jedoch nicht. Der Staat erklärt den Animismus für primitiv und versucht, ihn zugunsten des Buddhismus auszumerzen. Allerdings hat nicht nur jede linguistische Gruppe, sondern vielfach jede Dorfgemeinschaft ein eigenes animistisches Universum. Wo endet, wo beginnt die Ethnie oder die Religion? Diese Frage verweist darauf, dass Ethnizität ebenso ein westliches Konstrukt ist wie die Religion.

Im Übrigen ist Laos auch ein gutes Beispiel gegen die zentrale These des Buches. Eine ausgeprägte ethnische Heterogenität geht mit sehr geringer Einkommensungleichheit einher. Der Autor würde argumentieren, wie er es am Beispiel Chinas tut, dass der Sozialismus dafür verantwortlich sei, also eine Randbedingung innerhalb seines Modells. Das Argument ist nicht ganz falsch, aber es muss mit dem ländlichen Charakter der Ökonomie und dem Vietnamkrieg verknüpft werden, der das Land dem Ausdruck Kissingers zufolge „in die Steinzeit zurück“ bombardierte. Das Faktorengeflecht ist so komplex, dass für jede Allgemeinaussage Gegenbeispiele gefunden werden können. Darin liegt eine der Schwächen von Webers Ansatz.

Warum aber steigt die Einkommensungleichheit in Laos und China in den letzten Jahren stark an? Der Autor erwähnt beiläufig einen Faktor, der interessanter Weise ansonsten im Buch fehlt, nämlich den Kapitalismus. Es ist erstaunlich, dass ein Buch über Einkommensungleichheit im internationalen Vergleich ohne eine Diskussion der Globalisierung des Kapitalismus auskommt. In diesem Zusammenhang ist auch die Vernachlässigung des Kolonialismus zu sehen. Haben Weltbank und IWF, Großunternehmen und Großkapital, die Kommodifizierung und Ausbeutung aller Ressourcen nichts mit dem Thema des Buches zu tun? Diese Faktoren brechen zwar an einigen Stellen in die Argumentation ein, aber sie werden nicht systematisch behandelt, sondern als gegeben vorausgesetzt. Ich halte Kolonialismus und Kapitalismus für die wichtigsten der erklärenden Faktoren. Die Ergebnisse des Buches sind mit unserer Erklärung sozialer Ungleichheit vereinbar (Rehbein / Souza, 2014), aber sie erlauben es leider nicht, sie kritisch zu hinterfragen.

Das Buch scheint eine problematische politische Schlussfolgerung nahe $\mathrm{zu}$ legen. Wenn ethnische Heterogenität Einkommensungleichheit verstärkt, müsste man die Homogenisierung verstärken. So will der Autor sein Werk jedoch nicht verstanden wissen. Wie bei Weber mischt sich eine konservative modernisierungsoptimistische Seite mit Zivilisationskritik. Im Schlusskapitel bezieht der Autor klar für Heterogenität Position, indem er nicht die Assimilation von Min- 
derheiten fordert, sondern ihre Stärkung. Dazu diskutiert er mehrere politische Maßnahmen, die zweifellos weiterführen. Dieses letzte Kapitel ist auch das einzige, das ich für die Lehre verwenden würde.

Vor dem Hintergrund der Kritik an Ausbeutung und Unterdrückung sowie des Schlusskapitels erscheint der erste Teil des Buches übrigens in einem anderen Licht. Man erkennt nicht nur die konservative Weberinterpretation von Albert und Schluchter, sondern auch die kritische von Habermas und Adorno (die allerdings im Buch nicht namentlich erwähnt werden). Wirkt das Buch zunächst, als wolle es nur weiteres Material für fragwürdige Maßnahmen von internationalen Organisationen und Regierungen liefern, widerlegt der Autor diesen Eindruck nach und nach. Am Ende geht es in eine interessante politische Richtung. Ferner gibt es zahlreiche Anregungen für die weitere Forschung zum Thema.

\section{Literatur}

Banerjee-Dube, I., Ed. Caste in History; Oxford University Press: New Delhi, 2008.

Jodhka, S. S. Caste; Oxford University Press: Neu-Delhi, 2013.

Jodhka, S. S.; Rehbein, B.; Souza, J. Inequality in Capitalist Societies; Routledge: London/ Singapore, 2017 (im Druck).

Rehbein, B.; Souza, J. Ungleichheit in kapitalistischen Gesellschaften; Beltz Juventa: Weinheim, 2014.

Shah, G., Ed. Dalits and the State; Concept Publishing: New Delhi, 2002.

Shah, G. et al. Untouchability in Rural India; Sage: New Delhi/London/Thousand Oaks, 2006. 